\title{
Fokker-Planck Simulation Study of Hot-Tail Effect on Runaway Electron Generation in ITER Disruptions*)
}

\author{
Hideo NUGA, Akinobu MATSUYAMA ${ }^{1)}$, Masatoshi YAGI ${ }^{1)}$ and Atsushi FUKUYAMA \\ Kyoto University, Kyoto-Daigaku-Katsura, Nisikyo-Ward, Kyoto 615-8540, Japan \\ 1) Japan Atomic Energy Agency, 2-166 Omotedate-Obuchi, Rokkasho 039-3212, Japan
}

(Received 30 November 2015 / Accepted 1 February 2016)

\begin{abstract}
A Fokker-Planck code TASK/FP, which calculates the evolution of the relativistic momentum distribution function of electrons and the induced toroidal electric field, has been applied to the study of runaway electron (RE) generation in ITER disruptions. The hot-tail effect on the RE generation is investigated. Hot tail is formed during the thermal quench ahead of the Dreicer generation, which have an impact on the subsequent current and the electric field evolution. The hot-tail affects the RE current density profile even in the secondary RE dominant case. In spite of the small ratio of the primary electrons, they are multiplied significantly by the avalanche effect. Consequently, the hot-tail effect plays an important role in determining the total RE current density profile after the current quench.
\end{abstract}

(C) 2016 The Japan Society of Plasma Science and Nuclear Fusion Research

Keywords: runaway electron, disruption, ITER, hot-tail effect, thermal quench

DOI: $10.1585 /$ pfr. 11.2403023

\section{Introduction}

The disruption is one of the most serious phenomena in tokamaks, which induces the huge electromagnetic forces to the device and generates the high-energetic runaway electrons (REs) causing the damage of plasma facing component [1]. In ITER, the RE current is considered to reach a few mega-amperes. If the thermal quench is sufficiently fast, the primary RE generation rate is enhanced through the so-called "hot-tail effect" $[2,3]$. In order to predict the disruption dynamics in ITER, kinetic FokkerPlanck calculations are required by taking into account of RE generation processes including the hot-tail effect.

The aim of this paper is to compare the RE generation process, especially RE current density profile, including and excluding the hot-tail effect in ITER. For this purpose, the Fokker-Planck code, named TASK/FP [4], is applied to simulations of the evolution of the relativistic electron momentum distribution function, the RE current, and the induced electric field in a self-consistent manner. The code treats the electron distribution function in twodimensional momentum space for sub-relativistic region (less than $0.5 \mathrm{MeV}$ ). In the present calculation, although the treatment of relativistic REs is simplified, the thermal and sub-relativistic electron distribution function is analyzed with a good resolution, being useful for detailed calculation of the primary RE generation rate.

As discussed above, the RE generation in a highcurrent tokamak like ITER is expected to be dominated by the secondary generation. Nevertheless, our simulation

author'se-mail: nuga@p-grp.nucleng.kyoto-u.ac.jp

*) This article is based on the presentation at the 25th International Toki Conference (ITC25). shows that the hot-tail formation plays an important role in determining the RE current profile after the current quench. Since even a small increment of the seed current density is amplified by the avalanche effect, the secondary RE current density profile is modified by the hot-tail effect.

In the rest of this paper, the following contents are discussed. Models used in our code are explained in Sec. 2. The numerical results of RE generation with an ITER-like parameters are shown in Sec.3. Conclusion and Discussion of this paper is provided in Sec. 4.

\section{Models}

In this paper, we evaluate the RE generation rate by using the kinetic Fokker-Planck code TASK/FP, which is two dimensional in momentum space and one dimensional in the minor radius. The equation can be written in the conservative form:

$$
\begin{aligned}
\frac{\partial f}{\partial t} & =-\nabla \cdot \boldsymbol{S}_{\mathrm{C}, \mathrm{E}} \\
& =-\nabla \cdot\left[-\stackrel{\leftrightarrow}{\boldsymbol{D}}_{\mathrm{C}} \cdot \nabla f+\left(\boldsymbol{F}_{\mathrm{C}}+\frac{q_{\mathrm{e}} E}{m_{\mathrm{e}}}\right) f\right],
\end{aligned}
$$

where $\nabla$ is the derivative operator in the momentum space $(p, \theta)$, and the subscripts $\mathrm{C}$ denotes the collision term. $p$ and $\theta$ denote the momentum and the pitch angle. In Eq. (1), the collision coefficients $\stackrel{\boldsymbol{D}}{\mathrm{C}}_{\mathrm{C}}$ and $\boldsymbol{F}_{\mathrm{C}}$ are determined with the weak relativistic isotropic background collision model $[5,6]$. It is assumed that the momentum distribution functions of the background plasma are Maxwellian for both electrons and ions.

In TASK/FP, the induced electric field is described by 
the following equations:

$$
\begin{aligned}
& \frac{1}{r} \frac{\partial}{\partial r}\left(r \frac{\partial E}{\partial r}\right)=\mu_{0} \frac{\partial}{\partial t} j, \\
& j=\sigma_{\|} E+e c\left(n_{\mathrm{rp}}+n_{\mathrm{rs}}\right), \\
& \frac{\mathrm{d} n_{\mathrm{rp}}}{\mathrm{d} t}=\int \nabla \cdot \boldsymbol{S}_{\mathrm{C}, \mathrm{E}} \mathrm{d} \boldsymbol{p}=-\int \frac{\partial f}{\partial t} \mathrm{~d} \boldsymbol{p}, \\
& \frac{\mathrm{d} n_{\mathrm{rs}}}{\mathrm{d} t}=n_{\mathrm{r}} \frac{E / E_{\mathrm{C}}-1}{\tau_{\mathrm{r}} \ln \Lambda} \sqrt{\frac{\pi \varphi}{3\left(Z_{\mathrm{eff}}+1\right)}} \times \\
& \left(1-\frac{E_{\mathrm{C}}}{E}+\frac{4 \pi\left(Z_{\mathrm{eff}}+1\right)^{2}}{3 \varphi\left(Z_{\mathrm{eff}}+1\right)\left(E^{2} / E_{\mathrm{C}}^{2}+4 / \varphi^{2}-1\right)}\right)^{-1 / 2},
\end{aligned}
$$

where $\sigma_{\|}$is the Spitzer conductivity $\sigma_{\text {sp }}=1.96 n_{\mathrm{e}} q_{\mathrm{e}}^{2} \tau_{\mathrm{e}} / m_{\mathrm{e}}$ $\left(\sim 35.7 T_{\mathrm{e}}^{3 / 2} / Z_{\mathrm{eff}}\left[\mathrm{MA} / \mathrm{V} \cdot \mathrm{m}, T_{\mathrm{e}}\right.\right.$ in $\left.\left.\mathrm{keV}\right]\right)$ with the neoclassical correction [7], $\tau_{\mathrm{e}}=3(2 \pi)^{3 / 2} \epsilon_{0}^{2} m_{\mathrm{e}}^{1 / 2} T_{\mathrm{e}}^{3 / 2} / n_{\mathrm{e}} q_{\mathrm{e}}^{4} \ln \Lambda$, $\tau_{\mathrm{r}}=4 \pi \varepsilon_{0}^{2} m^{2} c^{3} / n_{\mathrm{e}} e^{4} \ln \Lambda, \varphi=1-1.46 \varepsilon^{1 / 2}+1.72 \varepsilon$, and $\varepsilon$ is the inverse aspect ratio. $n_{\mathrm{rp}}$ and $n_{\mathrm{rs}}$ are the primary and secondary RE densities, and the critical electric field $E_{\mathrm{C}}=n_{\mathrm{e}} q_{\mathrm{e}}^{3} \ln \Lambda / 4 \pi \epsilon_{0}^{2} m_{\mathrm{e}} c^{2}\left(\sim 9.17 \times 10^{-22} n_{\mathrm{e}}\left[\mathrm{V} / \mathrm{m}, n_{\mathrm{e}}\right.\right.$ in $\left.\mathrm{m}^{-3}\right]$ ), respectively. Equation (2) expresses the electric field diffusion and this equation is closed by the Ohm's law (Eq. (3)), where we assume that the current density consists of two components: the bulk current, $\sigma_{\|} E$, and the RE current, $e c n_{\mathrm{r}}$. The boundary condition for the electric field is obtained by the expression of $E$ in vacuum region: $E(r) \propto \ln (r / b)$ and $E(r=b)=0$, where $b$ is the location of the wall. The total RE density $n_{\mathrm{r}}$ is evaluated from the sum of the primary $\left(n_{\mathrm{rp}}\right)$ and secondary $\left(n_{\mathrm{rs}}\right)$ REs; they are evaluated from Eqs. (4) and (5), respectively. In Eq. (4), the rate of the primary RE generation, which is the seed generation for the subsequent secondary generation, is given by calculating the flux through the boundary of momentum calculation domain: $0<p<p_{\max }$, where $\left(p_{\max }\right)^{2} / 2 m_{\mathrm{e}}$ $\sim 0.5 \mathrm{MeV}$. This means that the electrons whose momentum is greater than $p_{\max }$ are regarded as REs. Because our research focuses on not the RE energy distribution but the number of REs generated in a disruption, the evolution of $f$ in the $p>p_{\max }$ region is not calculated. By the same token, we assume that REs travel with the velocity of light in order to simplify the treatment of relativistic REs. The rate of the secondary RE generation $\mathrm{d} n_{\mathrm{rs}} / \mathrm{d} t$ [8], Eq. (5), which is caused by the avalanche mechanism, is given by a function of the RE density $n_{\mathrm{r}}$ and the electric field normalized by the critical electric field $E / E_{\mathrm{C}}$ rather than the momentum distribution. This is because the secondary RE generation rate is insensitive to the primary RE energy [9].

To calculate the conductivity, $\sigma_{\|}$, and the collision coefficients, $\stackrel{\boldsymbol{D}}{\mathrm{C}}_{\mathrm{C}}$ and $\boldsymbol{F}_{\mathrm{C}}$, requires the background plasma temperature, therefore, the evolution of the plasma temperature being given as a function of time. Here, the decay of the plasma temperature is modeled with the time constant of the thermal quench $\tau_{\mathrm{q}}$ :

$$
\begin{aligned}
& T(t, \rho)=\left(T_{0}(\rho)-T_{\mathrm{f}}(\rho)\right) \exp \left(-t / \tau_{\mathrm{q}}\right)+T_{\mathrm{f}}(\rho), \\
& T_{0}(\rho)=\left(T_{0}(0)-T_{0}(1)\right)\left(1-\rho^{2}\right)^{2}+T_{0}(1), \\
& T_{\mathrm{f}}(\rho)=T_{\mathrm{f}}(0)\left(1-0.9 \rho^{2}\right),
\end{aligned}
$$

Table 1 Plasma parameters employed in this paper [2,12].

\begin{tabular}{|l|l|}
\hline Radii & $R=6.2 \mathrm{~m}, a=2.0 \mathrm{~m}, b=2.4 \mathrm{~m}$ \\
\hline Initial current & $15 \mathrm{MA}$ \\
Current density & $j_{0}\left(1-\rho^{1.74}\right)^{3.23}$ \\
\hline Initial temperature & $T_{0}(0)=22.7 \mathrm{keV}, T_{0}(1)=2.27 \mathrm{keV}$ \\
Post-quench temp. & $T_{\mathrm{f}}(0)=10 \mathrm{eV}$ \\
\hline Density profile & $n(\rho)=(n(0)-n(1))\left(1-\rho^{2}\right)^{0.67}+n(1)$ \\
& $n(0)=1.06 \times 10^{20} \mathrm{~m}^{-3}$ \\
& $n(1)=1.06 \times 10^{19} \mathrm{~m}^{-3}$ \\
\hline Effective charge & $Z_{\text {eff }}=1$ \\
Ion species & Deuteron \\
\hline
\end{tabular}

where $\rho$ denotes the normalized minor radius, whereas $T_{0}$ and $T_{\mathrm{f}}$ are the initial and post-quench temperature, respectively. In Fokker-Planck simulations, electrons and ions are assumed to have the same temperature.

For simulations, we choose ITER-like parameters tabulated in Table 1. The simulations were performed with the number of the grids $\left(N_{\mathrm{p}}, N_{\theta}, N_{\mathrm{r}}\right)=(1024,64,32)$ and it was decomposed into 1024 cores by Message Passing Interface.

In the followings, in order to examine the effect of the hot-tail, comparisons have been made for the results including and excluding the hot-tail effect. The results excluding the hot-tail effect are obtained by the simulation which uses the theoretical RE generation rate model for Dreicer generation rate [10] instead of Eq. (4). The expression of the theoretical model is given by:

$$
\begin{aligned}
\frac{1}{n_{\mathrm{e}}} \frac{\mathrm{d} n_{\mathrm{rp}}}{\mathrm{d} t}= & C_{\mathrm{R}}\left(\alpha, Z_{\mathrm{eff}}\right) \hat{E}^{-h\left(\alpha, Z_{\mathrm{eff}}\right)} \\
& \times \exp \left(-\frac{\lambda(\alpha)}{4 \hat{E}}-\sqrt{\frac{2}{\hat{E}} \Gamma\left(\alpha, Z_{\mathrm{eff}}\right)}\right),
\end{aligned}
$$

where, $\hat{E}=E / E_{\mathrm{D}}, \alpha=\hat{E} m c^{2} / T$,

$$
\begin{aligned}
& h\left(\alpha, Z_{\mathrm{eff}}\right)=\frac{1}{16(\alpha-1)}\left[\alpha\left(Z_{\mathrm{eff}}+1\right)-Z_{\mathrm{eff}}+7\right. \\
& \left.+2 \sqrt{\frac{\alpha}{\alpha-1}}\left(1+Z_{\mathrm{eff}}\right)(\alpha-2)\right], \\
& \lambda(\alpha)=8 \alpha\left(\alpha-\frac{1}{2}-\sqrt{\alpha(\alpha-1)}\right), \\
& \Gamma\left(\alpha, Z_{\mathrm{eff}}\right)=\sqrt{\frac{\left(1+Z_{\mathrm{eff}}\right) \alpha^{2}}{8(\alpha-1)}}\left[\frac{\pi}{2}-\arcsin \left(1-\frac{2}{\alpha}\right)\right],
\end{aligned}
$$

and $C_{\mathrm{R}}\left(\alpha, Z_{\mathrm{eff}}\right)$ is an unknown constant of order unity in $\varepsilon$. The unknown constant $C_{\mathrm{R}}$ is chosen to be 0.35 for the following calculation. This value is conventionally used in RE simulations [11].

\section{Results}

The hot-tail effect becomes effective when the thermal quench time is shorter than the electron-electron slowing down time for a few times of the initial thermal velocity 


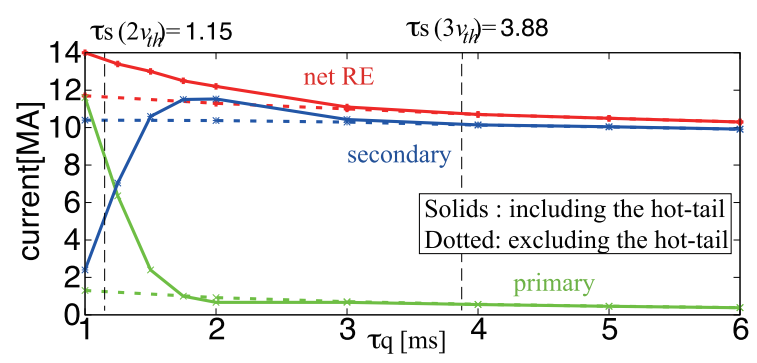

Fig. 1 RE currents versus thermal quench time are shown. Solid and dotted curves denote the RE currents including and excluding the hot-tail effect. Red, green, and blue curves are the net RE, the primary RE, and the secondary RE current, respectively.

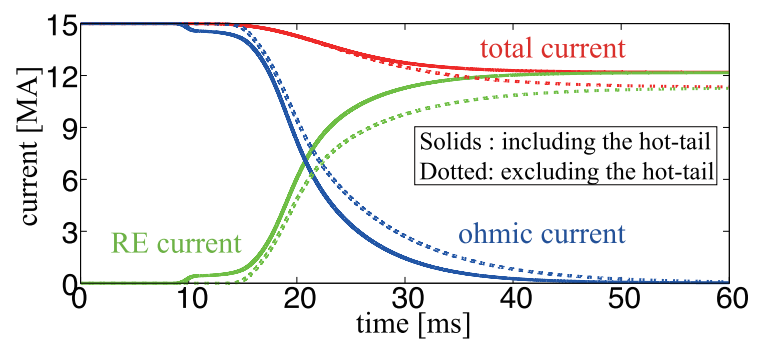

Fig. 2 Evolutions of total, RE, and ohmic current for $\tau_{\mathrm{q}}=$ $2.0 \mathrm{~ms}$. Solids and dotted curves denote the results including and excluding the hot-tail effect.

[4]. The e-e slowing down time is given by:

$$
\tau_{\mathrm{s}}^{e e}(v)=0.5 \frac{4 \pi \epsilon_{0}^{2} m_{\mathrm{e}}^{2} v^{3}}{n_{\mathrm{e}} q^{4} \ln \Lambda} .
$$

Figure 1 shows the relation between the thermal quench time to generated RE currents after the steady state is reached with RE current plateau in the simulations. The horizontal axis denotes the thermal quench time and the vertical axis denotes the amount of the RE current, where the total, primary, and secondary currents are plotted. Dashed vertical lines show the e-e slowing down time calculated with the test-particle velocity of $2 v_{\text {th }}$ and $3 v_{\text {th }}$. From Fig. 1, it is found that when $\tau_{\mathrm{q}}$ becomes shorter than $\tau_{\mathrm{s}}\left(3 v_{\text {th }}\right)$, the net RE current becomes larger than those calculated excluding the hot-tail effect. The primary RE current is seen to be comparable with the secondary RE current when $\tau_{\mathrm{q}}$ is shorter than $2 \mathrm{~ms}$. Because the RE current is generated to compensate the poloidal magnetic flux during the resistive decay of the ohmic current, the secondary RE current generation becomes less pronounced for the case where the primary RE current reaches the magnitude large enough to maintain the electric field below $E_{\mathrm{C}}$. Figure 2 shows the evolutions of total, RE, and ohmic current for $\tau_{\mathrm{q}}=2.0 \mathrm{~ms}$ in Fig. 1. It is found that the generation of RE current and the decay of the ohmic current are triggered earlier owing to the hot-tail effect. This is because hot-tail electrons can become REs easily even with the weak electric field due to the low collisionality.

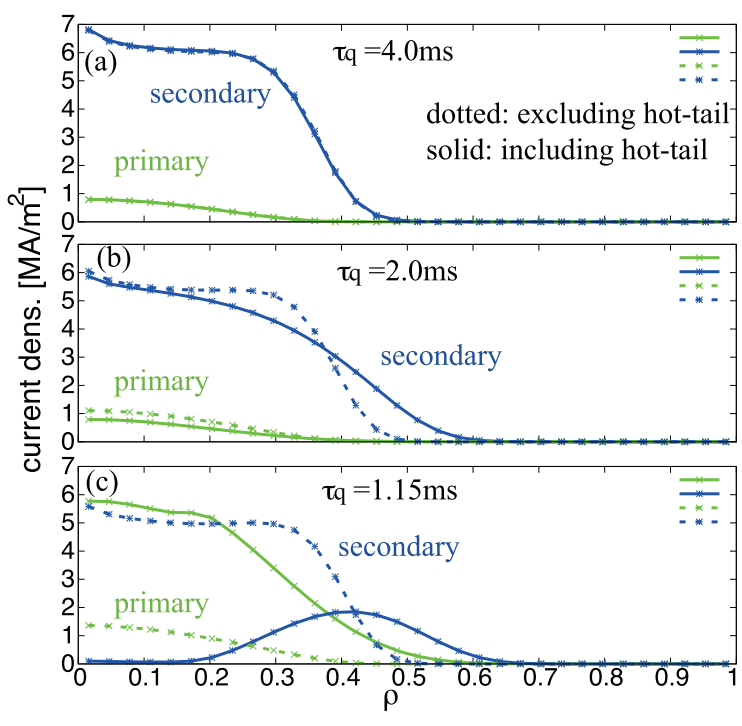

Fig. 3 Current density profiles for $\tau_{\mathrm{q}}=4.0 \mathrm{~ms}, 2.0 \mathrm{~ms}$, and 1.15 $\mathrm{ms}$ are shown. Solid and dotted curves denote the RE current density including and excluding the hot-tail effect. Green and blue curves are the primary and secondary RE current density at the time when the induce electric field vanishes after the current quench.

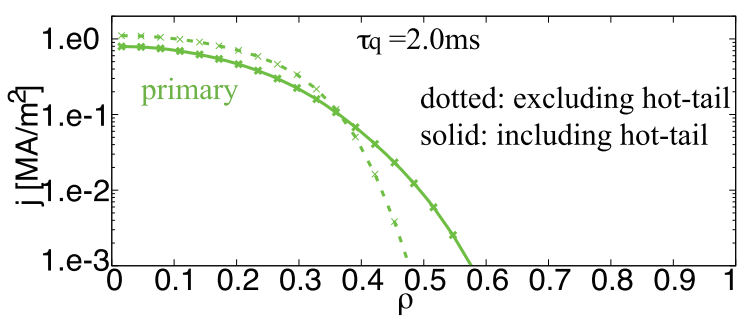

Fig. 4 The primary RE current density profiles for $2.0 \mathrm{~ms}$ in the logarithmic. Solid and dotted curves denote the RE current density including and excluding the hot-tail effect, respectively.

Figure 3 shows the RE current density profiles for (a) $\tau_{\mathrm{q}}=4.0 \mathrm{~ms}$, (b) $\tau_{\mathrm{q}}=2.0 \mathrm{~ms}$, (c) $\tau_{\mathrm{q}}=1.15 \mathrm{~ms}$, respectively. From Fig. $3(\mathrm{a})$, when $\tau_{\mathrm{q}}$ is longer than $\tau_{\mathrm{s}}\left(3 v_{\text {th }}\right) \simeq 3.88 \mathrm{~ms}$, the primary and the secondary RE current density profiles are in good agreement to those excluding the hot-tail effect. On the contrary, Fig. 3 (b) shows that the hot-tail effect changes not only the amount of RE current but also the RE current density profile, being broader in the case including the hot-tail generation. The enlargement of primary RE current density profiles are displayed in Fig. 4. We see that the hot-tail effect reduces the primary RE current density around magnetic axis and enhances it in outer region $(\rho>0.4)$.

Such changes of the RE current density profiles can be explained as follows. In Figs. 5 (b), (c), and (d) a small peak of the primary generation rate at $t \sim 10 \mathrm{~ms}$ corresponds to the hot-tail generated REs. Because of the highvelocity of the hot-tail electrons, they can be REs even with the weak electric field. Conversely, if the hot-tail ef- 


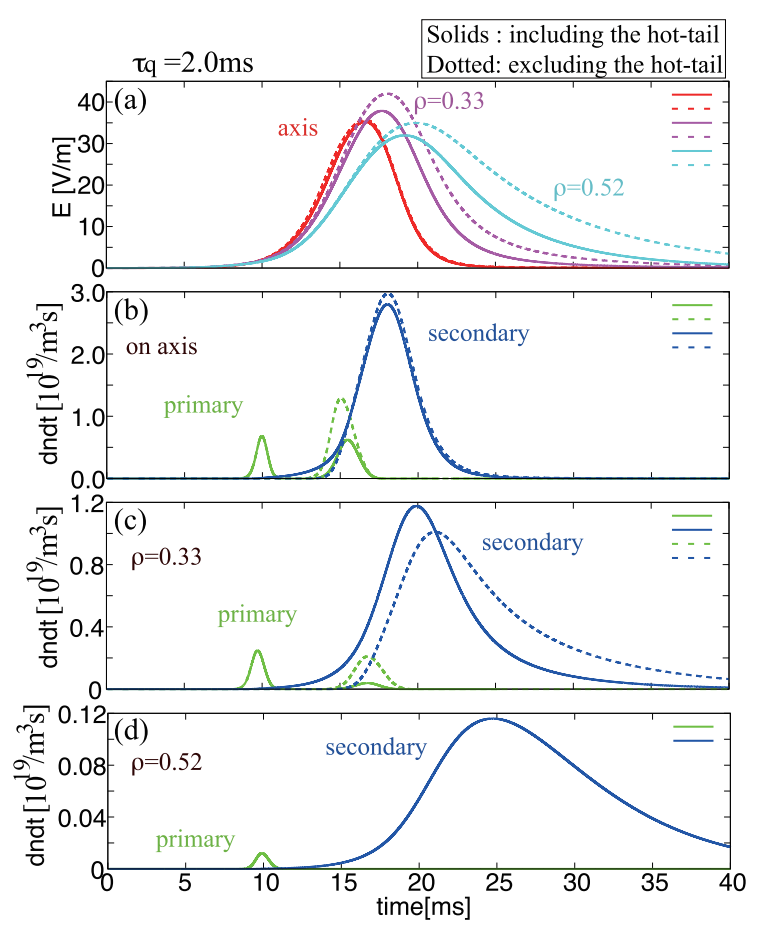

Fig. 5 Evolutions of (a) the induced electric field, (b) the RE generation rate on axis, (c) that on $\rho=0.33$, and (d) that on $\rho=0.52$ for $\tau_{\mathrm{q}}=2.0 \mathrm{~ms}$. In Fig. (d), the RE generation rates excluding the hot-tail effect are not displayed since they are negligibly small in this scale.

fect is excluded, the first peak of the primary RE generation rate is yielded at $t \sim 15 \mathrm{~ms}$ [Figs. 5 (b) and (c)]. The hot-tail REs are yielded at the earlier time than the Dreicer generation and they also trigger the secondary generation. Such an earlier onset on the secondary generation maintains the induced electric field weaker (in Fig. 5 (a)) to be consistent with the conservation of the poloidal flux. Hence the subsequent Dreicer generation at $t \sim 15 \mathrm{~ms}$ decreases since the Dreicer generation rate is sensitive to the electric field strength. As seen in Fig. 5(b), because the decrement of the second peak $(t \sim 15 \mathrm{~ms})$ is greater than the increment of the first peak $(t \sim 10 \mathrm{~ms})$, the primary REs slightly decrease on the axis. From a similar comparison, we see why the primary REs increase in the outer region $(\rho>0.4)$. Finally, small changes of the primary RE profile in Fig. 4 is amplified by the avalanche effect, and the secondary RE current density profile is modified as illustrated in Fig. 3 (b).

Figure 3 (c) illustrates the calculated RE current density profile for $\tau_{\mathrm{q}}=1.15 \mathrm{~ms}$, where the hot-tail effect dominates RE generation on-axis. In this case, the primary $\mathrm{RE}$ current density is evaluated to be much larger than in the case excluding the hot-tail effect for the whole radial zone. In the inner radial zone $(\rho<0.2)$, the resistive decay of the poloidal magnetic flux is compensated solely by the primary RE generation rate owing to the high primary generation rate as shown in Fig. 6(b). This reduces the electric field around the axis to nearly zero (in Fig. 6(a)),

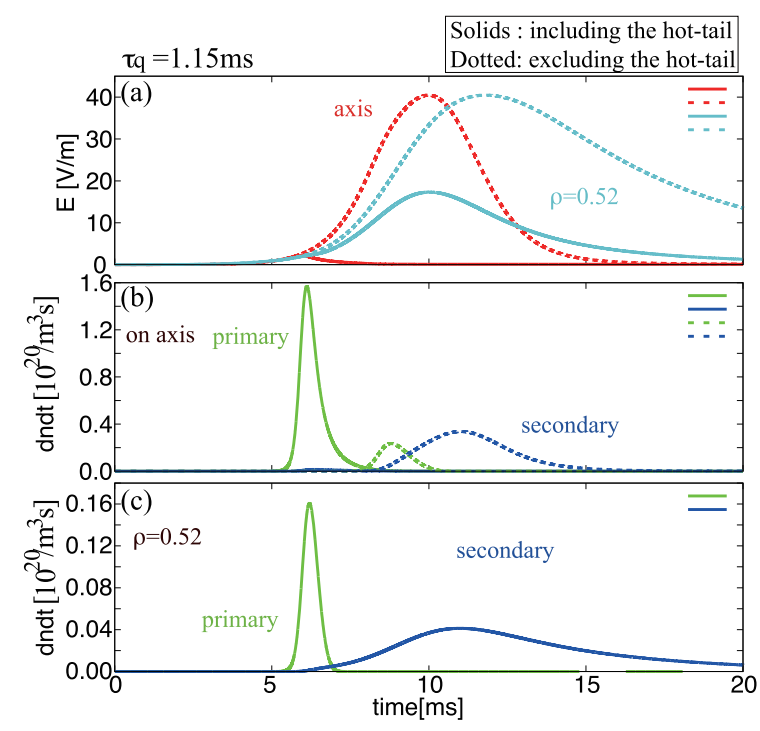

Fig. 6 Evolutions of (a) the induced electric field, (b) the RE generation rate on axis, and (c) that on $\rho=0.52$ for $\tau_{\mathrm{q}}=$ $1.15 \mathrm{~ms}$. In Fig. (c), the RE generation rates excluding the hot-tail effect are not displayed since they are negligibly small in this scale.

therefore the secondary generation being suppressed. On the contrary, in the outer region $(\rho \sim 0.5)$, the electric field is maintained enough to generate secondary REs (in Figs. 6(a) and (c)). Consequently, the secondary RE current density profile becomes hollow.

\section{Conclusion and Discussion}

We have applied the kinetic Fokker-Planck simulation code TASK/FP to describe the evolution of electron distribution function during the RE generation in ITERlike plasmas including the hot-tail effects. The hot-tail becomes important when the thermal quench time $\tau_{\mathrm{q}}$ is shorter than the typical slowing time of electrons with a few times of the initial thermal velocity $\tau_{\mathrm{s}}^{e e}\left(2-3 v_{\text {th }}\right)$. We have confirmed that, if the thermal quench is sufficiently faster than the condition, the primary RE current carried by the hot-tail electrons becomes larger than the secondary RE current generated by avalanche effect. We have found that the hot-tail effect also strongly affects the radial profile of RE current density.

Since the generation of hot-tail electrons, inductive toroidal electric field, and secondary electrons are strongly coupled with each other, careful analyses are required for quantitative prediction of RE generation. For the mitigation of RE generation, massive gas injection (MGI) has been proposed and tested in tokamak plasmas, since MGI is expected to increase the plasma density and $Z_{\text {eff }}$ and to suppress the generation of REs by high collisionality. If MGI also shorten the thermal quench time, however, the hot-tail effect may enhance the primary RE generation and nullify the mitigation of REs. The analysis MGI is left for future study including the modeling of thermal quench. 
[1] T.C. Hender et al., Nucl. Fusion 47, S128 (2007).

[2] H. Smith and E. Verwichte, Phys. Plasmas 15, 072502 (2008).

[3] T. Fehér, H. Smith, T. Fülöp et al., Plasma Phys. Control. Fusion 53, 035014 (2011).

[4] H. Nuga, A. Matsuyama et al., J. Plasma Fusion Res. 10, 1203006 (2015).

[5] C.F.F. Karney and N.J. Fisch, Phys. Fluids 28, 116 (1985).

[6] C. Karney, Computer Physics Reports 4, 183 (1986).

[7] J. Wesson, Tokamaks Third edition, (Clarendon, Oxford,
2004) p.737.

[8] M.N. Rosenbluth and S.V. Putvinski, Nucl. Fusion 37, 1355 (1997).

[9] S. Chiu, M. Rosenbluth et al., Nucl. Fusion 38, 1711 (1998).

[10] J.W. Conner and R.J. Hastie, Nucl. Fusion 15, 415 (1975).

[11] R.M. Kulsrud et al., Phys. Rev. Lett. 31, 690 (1973).

[12] R. Aymar, P. Barabaschi and Y. Shimomura, Plasma Phys. Control. Fusion 44, 519 (2002). 